\title{
Cross-sectional small intestinal surveillance of maintenance hemodialysis patients using video capsule endoscopy: SCHEMA study
}

Authors

Institutions
Naoki Hosoe ${ }^{1}$, Shigeaki Matsukawa ${ }^{2}$, Yoshihiko Kanno ${ }^{3}$, Makoto Naganuma ${ }^{4}$, Hiroyuki Imaeda ${ }^{5}$, Yosuke Ida ${ }^{4}$, Yoshitsugu Tsuchiya ${ }^{2}$, Toshifumi Hibi ${ }^{4,6}$, Haruhiko Ogata ${ }^{1}$, Takanori Kanai ${ }^{4}$

Institutions are listed at the end of article. submitted 1. October 2015 accepted after revision 21. February 2016

\section{Bibliography}

Dol http://dx.doi.org/ 10.1055/s-0042-105203 Published online: 15.4.2016 Endoscopy International Open 2016; 04: E589-E596

(c) Georg Thieme Verlag KG Stuttgart . New York E-ISSN 2196-9736

\section{Corresponding author}

\section{Naoki Hosoe, MD, PhD}

Center for Diagnostic and

Therapeutic Endoscopy

School of Medicine

Keio University

35 Shinanomachi

Shinjuku, Tokyo 160-8582

Japan

Fax: +81-3-3357-2778

nhosoe@keio.jp
Background and study aims: Small intestinal pathology in hemodialysis (HD) patients has been studied in only a small number of retrospective case series. One method for noninvasively surveying small intestinal disorders is video capsule endoscopy (VCE). The primary aim of this study was to investigate the prevalence of small intestinal abnormalities among asymptomatic maintenance HD outpatients using VCE. The secondary aim was to assess the clinical impact of these abnormalities.

Patients and methods: This study consisted of two phases. In phase I, a cross-sectional study, a cohort of patients who received maintenance HD three times weekly at an outpatient hemodialysis clinic were studied using VCE. Phase II was a prospective cohort study with follow up for 1 year after VCE.

Results: Fifty-six patients were enrolled in this study, and two were excluded from analysis due

\section{Introduction}

The incidence and prevalence of end-stage renal disease (ESRD) have been increasing worldwide [1]. In Japan, the number of patients undergoing maintenance hemodialysis (HD) has increased annually, and more than 300000 patients received hemodialysis in 2012 [2]. A previous study reported that ESRD patients tend to have various gastrointestinal lesions and associated complications [3,4]. In 2012, $1.1 \%$ of annual deaths among Japanese HD patients were caused by intestinal obstruction [2]. Moreover, uremia leads to the tendency to bleed [3], and $1.7 \%$ of annual deaths among Japanese HD patients were caused by hemorrhage including gastrointestinal bleeding [2]. Several reports have shown that obscure gastrointestinal bleeding occurred more frequently in patients on hemodialysis [5,6]. Obscure gastrointestinal bleeding is difficult to treat, and can be fatal. Thus, investigation of the incidence and to capsule retention in the stomach. The prevalence of small bowel abnormalities in HD patients was $64.8 \%$ (35/54) (95\% confidential interval $52.1 \%-77.6 \%)$. Of 54 patients, 21 (38.9\%) had mucosal lesions, 10 (18.5\%) had vascular lesions, and 4 (7.4\%) had both lesion types. During the 1 -year follow-up period, events occurred in four patients. A small bowel-associated event was observed in one patient, who underwent laparoscopy-assisted small intestinal partial resection 3 months after diagnosis by VCE. All patients in whom events were seen had small bowel abnormalities; no events were observed in the VCE-negative group.

Conclusions: Although asymptomatic maintenance HD patients had a high prevalence of small bowel abnormalities (64.8\%), they did not have a high incidence of small bowel-associated events during the 1-year follow-up.

prevalence of small intestinal disease is crucial for patients undergoing maintenance HD. Several studies have reported the incidence and prevalence of upper gastrointestinal bleeding $[7,8]$ and lower gastrointestinal bleeding [9] in patients with ESRD; however, mid-gastrointestinal bleeding (small intestinal) pathology in patients with ESRD has been studied in only a limited number of retrospective case series [10-14].

Video capsule endoscopy (VCE) was first described in 2000 [15]. The VCE device is noninvasive and useful for detecting and diagnosing small bowel diseases such as obscure gastrointestinal bleeding [16,17], small bowel tumors [18], and inflammatory bowel disease [19]. For the VCE procedure, the patient simply swallows the capsule; its minimally invasive nature is well-accepted by patients. Thus, VCE is an appropriate device for the surveillance of small intestinal disease.

The primary aim of this prospective observational study was to investigate the prevalence of small 
intestinal abnormalities among asymptomatic maintenance HD outpatients using VCE. The secondary aim was to assess the clinical impact of these abnormalities on HD patients by following this cohort for 1year.

\section{Patients and methods}

$\nabla$

\section{Study design}

This study, named SCHEMA (Small intestinal surveillance of Chronic HEModialysis Asymptomatic patients), was approved by the ethics committee of Keio University Hospital, based on the Declaration of Helsinki and the STROBE statement [20], and registered through the University hospital Medical Information Network (UMIN) Clinical Trials Registry, which is approved by the International Committee of Medical Journal Editors (UMIN ID000003349). All enrolled patients were informed of the risks and complications of VCE, such as capsule retention. Written informed consent was obtained from all patients. The protocol was established in February 2010. Patient enrollment began in March 2010 and ended in July 2010. This study consisted of two phases. In phase I, a cross-sectional study, the interventional VCE device was used on a cohort of patients who were receiving maintenance HD. To avoid over-diagnosis of the detected lesions, a case-control analysis was also performed based on the results of VCE. Cases were defined as patients with positive findings detected by VCE, and controls were defined as patients with no positive findings on VCE. In phase II, which was a prospective cohort study, the cohort was followed for 1 year after VCE to assess the clinical impact of the small intestinal abnormalities that were detected with VCE. Events were defined as a hospital admission associated with small bowel disease or death from any cause. All events during the follow-up period were recorded.

\section{Study cohort}

The cohort consisted of asymptomatic outpatients who received HD three times per week at an outpatient hemodialysis clinic, the Keishinkai Tama Nagayama Jin-Naika Clinic in western Tokyo. Eligible patients had received HD for more than 3 months before enrollment. Excluded patients were those with contraindications to the small bowel capsule [21]. To prevent capsule retention, patients with a history of abdominal polysurgery (underwent surgery more than twice) or continuous ambulatory peritoneal dialysis were excluded. In the event of capsule retention, a patient requires surgery or invasive therapy. Therefore, to avoid operative risks, patients who had severe heart failure $(\geq \mathrm{New}$ York Heart Association [NYHA] III) and/or severe respiratory failure ( $\geq$ Hugh-Jones [H-J] IV), were excluded. Asymptomatic patients were defined as those with no history of melena or hematochezia within 3 months before enrollment.

\section{VCE procedure}

All VCE procedures were performed using the Pillcam SB device (Given Imaging Ltd, Tokyo, Japan) between March and July 2010 at the Keishinkai Tama Nagayama Jin-Naika Clinic. Patients swallowed the VCE device after a 12-hour fast with neither premedication nor bowel preparation. Drinking and eating were permitted at 2 and 4 hours after swallowing the video capsule, respectively. The recorded digital information was downloaded from the recorder into the workstation, and the images were analyzed using the proprietary RAPID 5 software by two experts (N.H. and Y.I.) who had each performed more than 150 VCE examinations.
These two readers were blinded to the clinical backgrounds of the patients in the VCE videos. All VCE findings were based on the consensus of the two experts. In the event of a discrepancy in the relevant findings, the differences were resolved by discussion until consensus was reached. To assess the gastrointestinal motility of HD patients, the gastric transit time, small bowel transit time, and whether the examination was completed (capsule reaching the cecum) were recorded as surrogate markers. Gastric transit time was defined as the time interval between the first gastric image and the first duodenal image. Small bowel transit time was defined as the time interval between the first duodenal image and the first cecal image. Completion rate was defined as the percentage of VCE procedures in which the capsule reached the cecum. Positive findings were categorized according to standard VCE terminology [22].

\section{Data collection}

Backgrounds and clinical data were collected by chart review from August 2010 to September 2010. Clinical background parameters included sex, age, duration of dialysis, etiology of ESRD, comorbidities and previous illnesses, and medications. Data on HD settings including dialysis time, administered dose of heparin preparations, administered dose of erythropoietin preparations, and administered iron preparations during the past 1 month were collected. Laboratory analyses of blood samples from all patients were performed every 2 weeks before and after HD. The most recent laboratory data (hemoglobin, ferritin, albumin, C-reactive protein (CRP), pre- and post-dialysis blood urea nitrogen, and pre- and post-dialysis creatinine) collected before the VCE procedure were used for analysis.

\section{Prospective cohort study}

Enrolled patients were followed for 1 year after VCE. Events were defined as a hospital admission associated with small bowel disease or death from any cause. All events during the follow-up period were recorded.

\section{Statistical analysis}

The data were analyzed using a per-protocol analysis. Statistical analysis was performed using One-way analysis of variance (ANOVA) for multiple group comparisons, and the Fisher's exact tests for categorical variables. $P$ values $<0.05$ were considered significant. SPSS version 19 software (IBM Armonk, NY USA) was used for all statistical analyses. All authors had access to the study data and reviewed and approved the final manuscript.

\section{Results}

$\nabla$

Between March 2010 and July 2010, 112 patients received HD at Keishinkai Tama Nagayama Jin-Naika Clinic. All patients were screened, and 19 were excluded. The study was explained to the remaining 93 patients, and 37 patients declined to participate. The remaining 56 patients were enrolled. In two patients, the capsule was retained in the stomach for 8 hours, and no small bowel pictures could be obtained. These two patients were excluded from analysis ( Fig. 1). Baseline demographic characteristics of the analyzed patients are shown in Table 1. The most common etiology of ESRD was diabetic nephropathy, and the mean duration of HD was 79.4 months. 


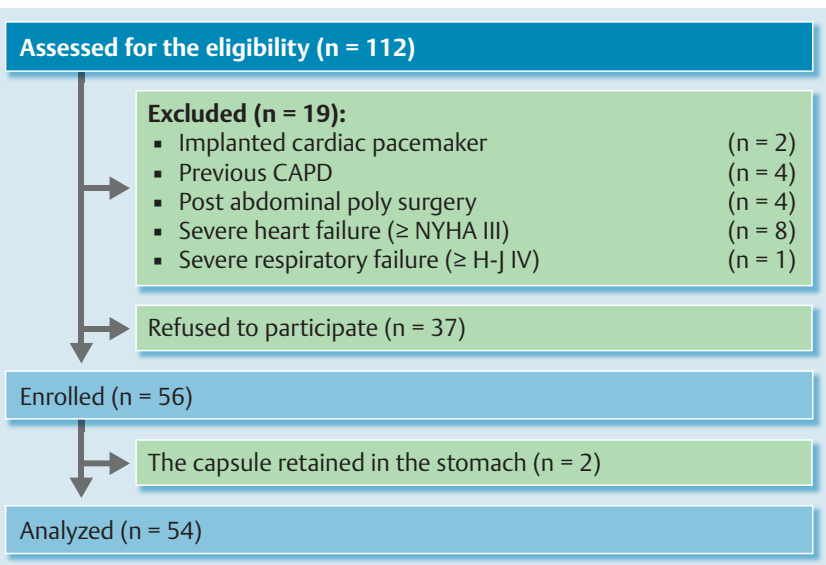

Fig. 1 Flow Diagram of the SCHEMA study. CAPD, continuous ambulatory peritoneal dialysis; NYHA, New York Heart Association; H-J, Hugh-Jones.

\section{Cross-sectional study and case-control study}

Excretion of the capsule was confirmed visually or by abdominal $\mathrm{X}$-ray, and no VCE-associated adverse events occurred in any of our cases. In the two patients who experienced capsule retention in the stomach for 8 hours, natural excretion of the capsule was confirmed within a few days without intervention. The prevalence of small bowel abnormalities in HD patients was $64.8 \%$ (35/54) (95\% confidential interval 52.1-77.6\%) ( $\odot$ Table2 and - Table3). Capsule findings could be classified into two categories: mucosal and vascular lesions. Mucosal lesions were subclassified as circular ulcer with stenosis ( $\mathbf{~ F i g . 2 ) , ~ e r o s i o n ~ ( ~} \bullet$ Fig.3) or small ulcer, or denuded mucosa ( $\bullet$ Table 2 ). Vascular lesions were subclassified as angioectasia ( $\bullet$ Fig. 4 ), small redness or petechia, or varix or hemangioma ( $\bullet$ Fig. 5 ) ( $\bullet$ Table 2). Of 54 patients, 21 (38.9\%) had mucosal lesions, 10 (18.5\%) had vascular lesions, and 4 (7.4\%) had both types of lesions ( $\bullet$ Table 3 and - Fig.3). The factors potentially associated with small intestinal abnormalities are shown in $\bullet$ Table 3 . No factors significantly differed between patients with and without positive VCE findings. We observed a trend toward a greater incidence of iron administration in the VCE positive-finding group compared to the negative group $(P=0.12)$.

\section{Transit time analysis}

In all patients, the total recoding time of the capsule was approxi-

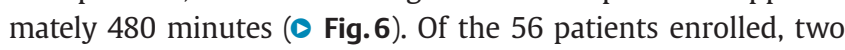
had a severe gastric motility disorder, one of whom also had diabetes. Furthermore, esophageal transit time greater than 30 minutes was observed in seven patients without esophageal stricture. Small bowel transit time ranged from 80 to 454 minutes. Among 54 patients, seven capsules could not reach the cecum within the battery life; thus the capsule completion rate was $87.0 \%(47 / 54)$.

\section{Prospective cohort study}

During the 1-year follow-up period, two patients were lost to follow-up, and events occurred in four patients ( $\bullet$ Table 4). A small bowel-associated event was observed in one patient: a circular ulcer with stenosis was detected by VCE ( $\boldsymbol{\bullet}$ Fig. 2 ) without capsule retention. That patient had been prescribed low-dose aspirin for arteriosclerosis obliterans and a nonsteroidal anti-inflammatory drug (NSAID) for lumbago. The patient was admitted after VCE and examined using balloon-assisted enteroscopy, which revealed a circular ulcer with severe stricture in the ileum $(\bullet$ Fig. 7). The patient received balloon dilation therapy via balloon-assisted enteroscopy twice. However, stricture and bloating remained. The patient opted for surgery and laparoscopy-assisted partial resection was performed 3 months after VCE. The pathological findings of the resected specimen showed an infiltration of inflammatory cells and fibrosis, with suspected ischemic enteritis and/or NSAID-induced enteropathy.

In another patient, a large hemangioma was identified by VCE in the jejunum ( $\bullet$ Fig.5) and subsequently performed balloon-as-

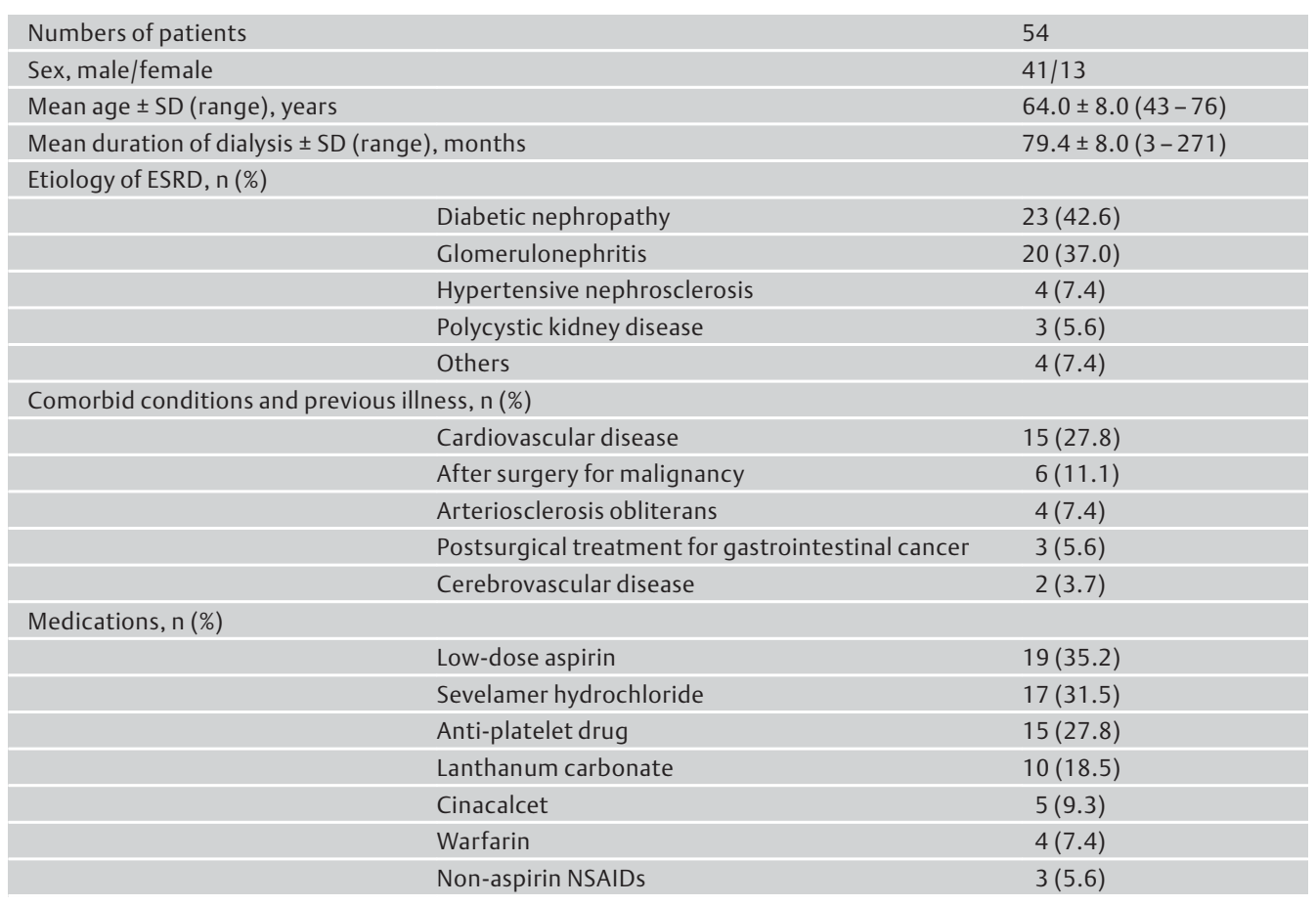

Table 1 Baseline Demographic Characteristics of Analyzed Patients.

SD, standard deviation; ESRD, end-stage renal disease; NSAIDs, nonsteroidal anti-inflammatory drugs. 
Table 2 Number of cases by type of capsule findings.

\begin{tabular}{|c|c|c|}
\hline & \multicolumn{2}{|c|}{ Capsule findings } \\
\hline & Mucosal lesion & Vascular lesion \\
\hline Angioectasia & & $9^{*}$ \\
\hline Small redness or petechia & & 2 \\
\hline Varix or hemangioma & & 3 \\
\hline Erosion or small ulcer & $22^{*}$ & \\
\hline Denuded mucosa & 2 & \\
\hline Circular ulcer with stenosis & 1 & \\
\hline
\end{tabular}

sisted enteroscopy showed similar findings ( $\bullet$ Fig. 8). That lesion was enteroscopically tattooed ( $\bullet$ Fig. 8). We suggested prophylactic surgery or follow-up monitoring, and the patient preferred to take a wait-and-see approach. During the follow-up period, no bleeding episodes occurred in that patient, thus that individual was not included in the event group. Death from any cause occurred in three patients. All events occurred in patients with small bowel abnormalities; no events were observed in the VCEnegative group.

Table 3 Factors and laboratory data for small intestinal abnormalities.

\begin{tabular}{|c|c|c|c|c|c|c|}
\hline \multirow[b]{2}{*}{ Factors } & \multirow[b]{2}{*}{ Number } & \multicolumn{5}{|l|}{ Capsule findings } \\
\hline & & Mucosal lesion & Vascular lesion & Vascular+mucosal lesion & None & $P$ value \\
\hline & 54 & $21(38.9 \%)$ & $10(18.5 \%)$ & $4(7.4 \%)$ & $19(35.2 \%)$ & \\
\hline \multicolumn{7}{|l|}{ Patients' background } \\
\hline \multicolumn{7}{|l|}{ Sex } \\
\hline male & 41 & $17(41.5 \%)$ & $7(17.1 \%)$ & $3(7.3 \%)$ & $14(34.1 \%)$ & 0.91 \\
\hline female & 13 & $4(30.8 \%)$ & $3(23.1 \%)$ & $1(7.7 \%)$ & $5(38.4 \%)$ & \\
\hline Age (years) & & $62.1 \pm 8.2$ & $63.6 \pm 9.6$ & $69.5 \pm 4.2$ & $65.2 \pm 7.2$ & 0.32 \\
\hline Duration of dialysis (months) & & $85.1 \pm 82.5$ & $85.1 \pm 60.4$ & $93.5 \pm 70.1$ & $67.1 \pm 64.9$ & 0.82 \\
\hline \multicolumn{7}{|l|}{ Etiology of ESRD } \\
\hline diabetic & 23 & $9(39.1 \%)$ & $3(13.0 \%)$ & $1(4.3 \%)$ & $10(43.5 \%)$ & 0.59 \\
\hline non-diabetic & 31 & $12(38.7 \%)$ & $7(22.6 \%)$ & $3(9.7 \%)$ & $9(29.0 \%)$ & \\
\hline \multicolumn{7}{|l|}{ Comorbidity } \\
\hline \multicolumn{7}{|l|}{ vascular disease } \\
\hline$(+)$ & 15 & $7(46.7 \%)$ & $3(20.0 \%)$ & $2(13.3 \%)$ & $3(20.0 \%)$ & 0.44 \\
\hline$(-)$ & 39 & $14(35.9 \%)$ & $7(17.9 \%)$ & $3(5.1 \%)$ & $16(41.0 \%)$ & \\
\hline \multicolumn{7}{|l|}{ Oral medication } \\
\hline \multicolumn{7}{|l|}{ Low-dose aspirin } \\
\hline$(+)$ & 19 & $8(42.1 \%)$ & $3(15.8 \%)$ & $3(15.8 \%)$ & $5(26.3 \%)$ & 0.30 \\
\hline$(-)$ & 35 & $13(37.1 \%)$ & $7(20.0 \%)$ & $1(2.9 \%)$ & $14(40.0 \%)$ & \\
\hline \multicolumn{7}{|l|}{ Sevelamer hydrochloride } \\
\hline$(+)$ & 17 & $6(35.3 \%)$ & $4(23.5 \%)$ & $2(11.8 \%)$ & $5(29.4 \%)$ & 0.73 \\
\hline$(-)$ & 37 & $15(40.5 \%)$ & $6(16.2 \%)$ & $2(5.4 \%)$ & $14(37.8 \%)$ & \\
\hline \multicolumn{7}{|l|}{ Anti-platelet drug } \\
\hline$(+)$ & 15 & $4(26.7 \%)$ & $6(40.0 \%)$ & $1(6.7 \%)$ & $4(26.7 \%)$ & 0.09 \\
\hline$(-)$ & 39 & $17(43.6 \%)$ & $4(10.3 \%)$ & $3(7.7 \%)$ & $15(38.5 \%)$ & \\
\hline \multicolumn{7}{|l|}{ PPI or H2RA } \\
\hline$(+)$ & 32 & $13(40.6 \%)$ & $4(12.5 \%)$ & $4(12.5 \%)$ & $13(40.6 \%)$ & 0.23 \\
\hline$(-)$ & 22 & $8(36.4 \%)$ & $6(27.3 \%)$ & $0(0.0 \%)$ & $8(36.4 \%)$ & \\
\hline \multicolumn{7}{|l|}{ Hemodialysis settings } \\
\hline Dialysis time/week (hours) & & $11.3 \pm 1.7$ & $10.7 \pm 1.3$ & $10.9 \pm 1.4$ & $10.0 \pm 1.2$ & 0.07 \\
\hline Dose of heparin/week (U) & & $10021.4 \pm 3298.8$ & $10920.0 \pm 4948.6$ & $8362.5 \pm 1012.7$ & $8897.4 \pm 2659.9$ & 0.19 \\
\hline Dose of EPO/week (IU) & & $3381.0 \pm 2970.3$ & $2650.0 \pm 2906.4$ & $3125.0 \pm 2926.2$ & $3079.0 \pm 2212.7$ & 0.92 \\
\hline \multicolumn{7}{|l|}{ Administered iron } \\
\hline$(+)$ & 8 & $6(75.0 \%)$ & $0(0.0 \%)$ & $0(0.0 \%)$ & $2(25.0 \%)$ & 0.12 \\
\hline$(-)$ & 46 & $15(31.6 \%)$ & $10(21.7 \%)$ & $4(7.4 \%)$ & $5(35.2 \%)$ & \\
\hline \multicolumn{7}{|l|}{ Laboratory data } \\
\hline Hemoglobin (g/dL) & & $10.5 \pm 0.8$ & $10.8 \pm 0.7$ & $11.3 \pm 0.8$ & $10.8 \pm 0.8$ & 0.23 \\
\hline Ferritin (ng/mL) & & $171.4 \pm 122.5$ & $164.1 \pm 129.6$ & $99.2 \pm 84.7$ & $193.5 \pm 138.6$ & 0.60 \\
\hline Albumin $(\mathrm{g} / \mathrm{dL})$ & & $4.0 \pm 0.3$ & $4.0 \pm 0.3$ & $4.2 \pm 0.2$ & $4.0 \pm 0.2$ & 0.54 \\
\hline $\operatorname{CRP}(\mathrm{mg} / \mathrm{dL})$ & & $0.3 \pm 0.4$ & $0.2 \pm 0.2$ & $0.1 \pm 0.1$ & $0.3 \pm 0.4$ & 0.58 \\
\hline \multicolumn{7}{|l|}{ BUN } \\
\hline pre-dialysis (mg/dL) & & $75.2 \pm 15.0$ & $79.2 \pm 11.6$ & $71.7 \pm 7.9$ & $72.2 \pm 16.0$ & 0.63 \\
\hline post-dialysis (mg/dL) & & $27.2 \pm 5.2$ & $29.0 \pm 6.9$ & $27.7 \pm 7.7$ & $26.9 \pm 6.4$ & 0.84 \\
\hline \multicolumn{7}{|l|}{ Creatinine } \\
\hline pre-dialysis (mg/dL) & & $12.3 \pm 2.6$ & $12.3 \pm 2.1$ & $10.3 \pm 3.1$ & $12.0 \pm 3.5$ & 0.66 \\
\hline post-dialysis (mg/dL) & & $5.1 \pm 1.3$ & $5.2 \pm 1.3$ & $4.4 \pm 1.1$ & $5.0 \pm 1.4$ & 0.72 \\
\hline
\end{tabular}

SD, standard deviation; ESRD, end-stage renal disease; PPI, proton pump inhibitor; H2RA, H2 receptor antagonist; EPO, erythropoietin; CRP, C-reactive protein; BUN, blood urea nitrogen. 


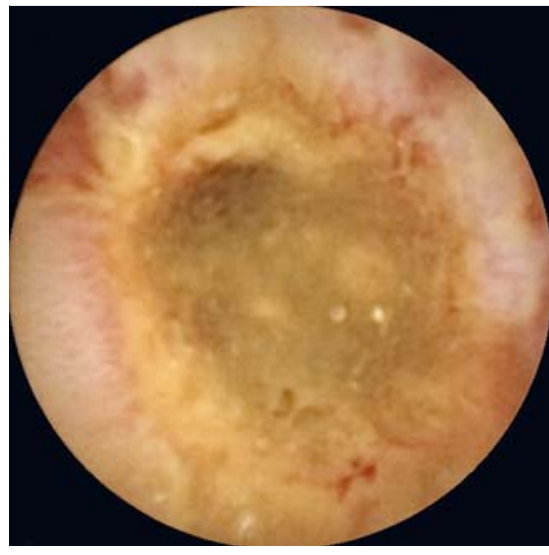

Fig. 2 Circular ulcer with stenosis detected by video capsule endoscopy.

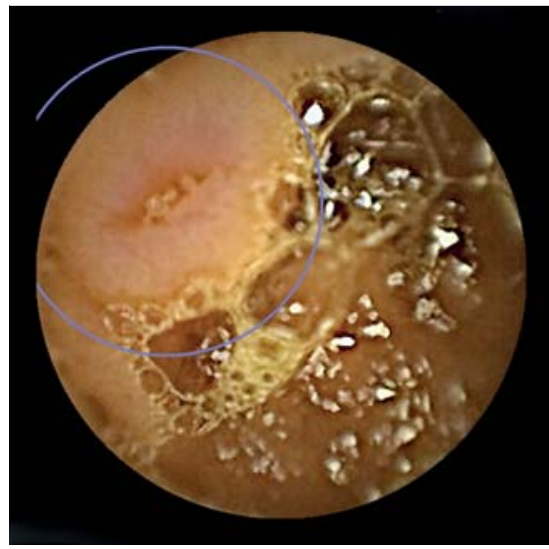

Fig. 3 Mucosal lesion (erosion) detected by video capsule endoscopy.

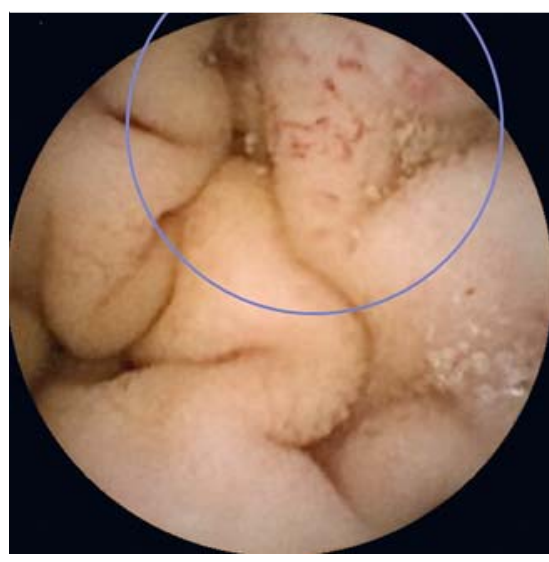

Fig. 4 Vascular lesion (angioectasia) detected by video capsule endoscopy.

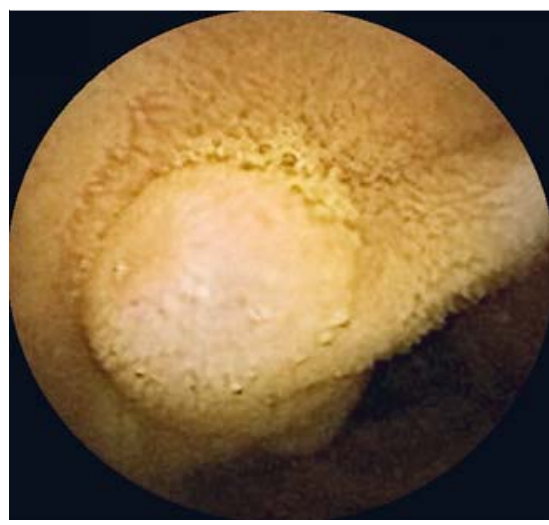

Fig. 5 Hemangioma detected by video capsule endoscopy.

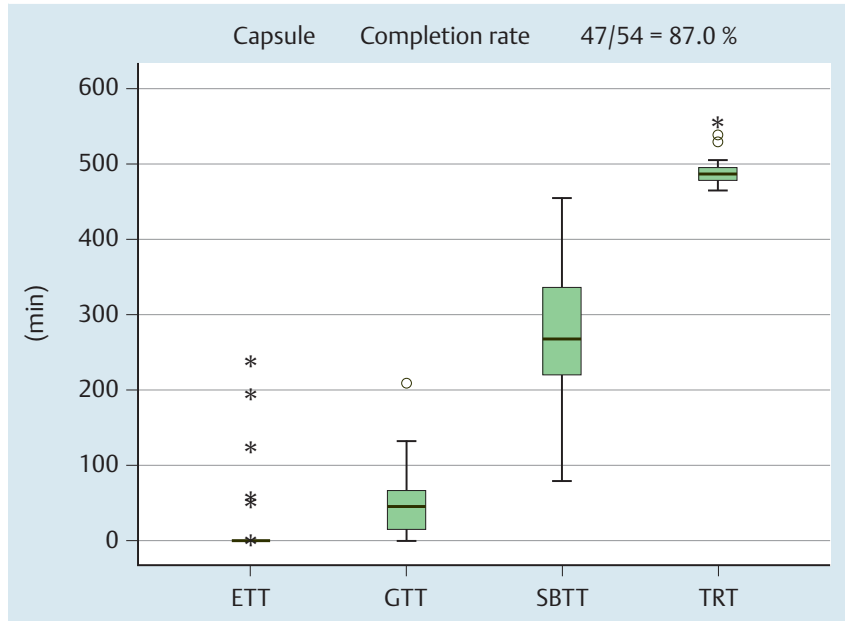

Fig. 6 Box plots of video capsule endoscopy transit time variables The box plots show the median values, interquartile ranges, and $95 \%$ ranges (extremes and outliers are not shown). ETT, esophageal transit time; GTT, gastric transit time; SBTT, small-bowel transit time; TRT, total recording time

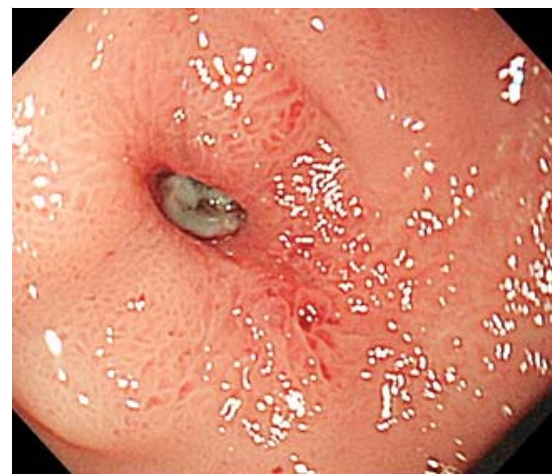

Fig. 7 Circular ulcer with stenosis detected by balloon-assisted enteroscopy.

\section{Discussion}

Seventy-five percent of patients with ESRD have gastrointestinal complaints such as nausea, vomiting, and anorexia [4]. Several previous studies have reported the incidence of upper gastrointestinal bleeding [23-27] and the prevalence of upper gastrointestinal bleeding [7] and lower gastrointestinal bleeding [9] disease among HD patients. By contrast, the incidence and prevalence of small intestinal disease in HD patients was unknown. Investigating the impact of small intestinal disease on HD is crucial for HD patients. This prospective cohort study is the first to investigate the prevalence of small intestinal disease among HD patients using VCE. The current study is a population-based survey of HD patients, and our results represent the varied clinical condition of HD patients. As shown in Table 1, the HD patients had various comorbidities, such as cardiovascular disease and cerebrovascular disease; various etiologies of ESRD, such as diabetic nephropathy and glomerulonephritis; and were taking various drugs, such as low-dose aspirin and NSAIDs. VCE provided images of small intestinal diseases that were not only related to renal failure, but also related to multiple factors such as diabetes, prescribed drugs, heart failure, and other comorbidities. The factors potentially associated with small intestinal abnormalities were analyzed in the current study, however no factors were significantly associated with small intestinal abnormalities. This suggests that many factors are related to small intestinal abnormalities. We observed a trend toward more prevalent administration 
Table 4 Outcome after 1-year follow-up.

\begin{tabular}{|lcccc}
\hline & $\begin{array}{l}\text { Capsule findings } \\
\text { Outcome }\end{array}$ & & & \\
\hline Mucosal lesion & Vascular lesion & Vascular+mucosal lesion & None \\
\hline Endoscopic therapy+operation & $1(1.9 \%)$ & $0(0.0 \%)$ & $0(0.0 \%)$ & $0(0.0 \%)$ \\
\hline Endoscopic investigation & $0(0.0 \%)$ & $1(1.9 \%)$ & $0(0.0 \%)$ & $0(0.0 \%)$ \\
\hline Other disease & & & & \\
\hline Death due to cerebral hemorrhage & $0(0.0 \%)$ & $1(1.9 \%)$ & $1(1.9 \%)$ & $0(0.0 \%)$ \\
\hline Death due to unknown disease & $1(1.9 \%)$ & $0(0.0 \%)$ & $0(0.0 \%)$ & $0(0.0 \%)$ \\
\hline Admission & $2(3.7 \%)$ & $2(3.7 \%)$ & $0(0.0 \%)$ & $0(0.0 \%)$ \\
\hline Missed follow-up & $1(1.9 \%)$ & $1(1.9 \%)$ & $0(0.0 \%)$ & $19(35.2 \%)$ \\
\hline No event & $16(29.5 \%)$ & $5(9.3 \%)$ & $3(5.5 \%)$ & $19(35.2 \%)$ \\
\hline Total & $21(38.9 \%)$ & $10(18.5 \%)$ & $4(7.4 \%)$ & \\
\hline
\end{tabular}

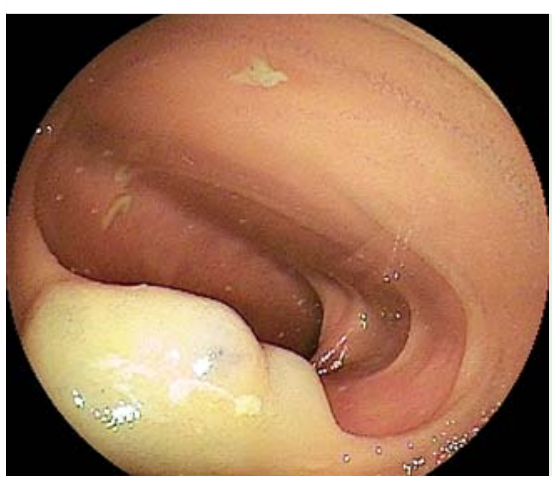

Fig. 8 Hemangioma detected by balloonassisted enteroscopy.

of iron in the VCE positive-finding group compared to the negative group $(P=0.12)$, whereas hemoglobin and ferritin levels did not differ between the groups. This may be because patients in our cohort underwent a blood examination every 2 weeks and received iron supplementation precisely according to their hemoglobin levels. One possible reason for the failure of our results to reach statistical significance may be the small size of the study, which may have been underpowered for detection of these associations. A larger cross-sectional clinical study is needed to confirm the observed absence of statistically significant differences. The prevalence of small bowel abnormalities in asymptomatic HD outpatients was $64.8 \%$. Graham et al. [28] reported that $71 \%$ of chronic NSAID users had small bowel injury detected by VCE, while $10 \%$ of a healthy control group had small intestinal mucosal injury. The prevalence of small intestinal disease among asymptomatic HD patients observed in the current study, like that in chronic NSAID users, was substantially higher than that observed in healthy controls.

In contrast to the present study, in which 21 of 54 patients (38.9 \%) had mucosal lesions, 10 (18.5\%) had vascular lesions, and 4 (7.4\%) had both lesion types, previous studies using retrospective analysis among symptomatic ESRD patients reported that vascular lesions were more often detected by VCE than were mucosal lesions $[10,11,13,14]$. Furthermore, the bleeding source of obscure gastrointestinal bleeding in HD patients was reported to be primarily vascular lesions such as angiodysplasia, varix, and hemangioma [6]. The discrepancy between these findings and the current results might be derived from differences between patient cohorts. Our results suggest that mucosal lesions are detected more often than vascular lesions in asymptomatic HD patients; however, overt bleeding was rare.
The clinical impact of VCE findings on asymptomatic HD was also evaluated in the follow-up phase. A small bowel-associated event was observed in one patient $(1 / 56,1.8 \%)$ in whom VCE detected a circular ulcer with stenosis without the development of severe abdominal symptoms. VCE enabled this patient to receive minimally invasive surgery. Screening for small intestinal disease on HD by VCE might lead to early detection of lesions requiring clinical intervention.

Interestingly, VCE results might influence patient outcomes. The VCE-negative group had no events over a 1-year period. As mentioned above, small intestinal abnormalities in HD patients might be caused by multiple factors such as uremia, NSAID and/or lowdose aspirin use, and microcirculatory disorders. Other studies have found that uremia can cause gastrointestinal mucosal abnormalities $[3,29]$; non-aspirin NSAID users had a higher incidence $(71 \%)$ of small intestinal mucosal injury [28]; non-aspirin NSAID use decreased the serum iron level of HD patients [30]; and low-dose aspirin can cause small intestinal mucosal injury [31 -33]. The small bowel abnormalities might be a reflection of multiple complicating disorders, demonstrating their impact on prognosis.

VCE may also reveal a patient's gastrointestinal motility function, using capsule transit time as a surrogate marker. A wireless motility capsule (SmartPill; Given Imaging Ltd, Yoqneam, Israel) measuring $26.8 \times 11.7 \mathrm{~mm}$ is used for assess gastrointestinal transit time and intestinal $\mathrm{pH}$ [34]. A wireless motility capsule is considered a useful diagnostic test for evaluating gastrointestinal motility [35]. However, the correlation between VCE transit time and gastrointestinal motility function is still unconfirmed. Thus, this was a limitation of the current transit time analysis. Furthermore, this analysis excluded two diabetic patients who had severe gastric motility disorders. The current results indicated that $3.6 \%(2 / 56)$ of patients had delayed gastric emptying and $12.5 \%$ (7/56) of enrolled patients had delayed esophageal emptying. Previous studies of abnormalities in gastric emptying in ESRD have reported conflicting results [4]. Although two patients had diabetes, VCE could directly measure the gastrointestinal transit time, which revealed the prevalence of delayed gastric emptying in HD patients. Francos et al. [36] reported that 4/56 (7\%) HD patients had manometric achalasia or diffuse esophageal spasm. Two other studies $[37,38]$ have suggested that asymptomatic motor disorders of the esophagus are likely to be present in ESRD. Although three of seven patients had diabetes, our data indicated that HD patients had esophageal motility disorders, as $12.5 \%$ of the capsules were retained in the esophagus. 
The current study had some limitations. Patients with severe heart and respiratory failure were excluded to decrease the risk of complications in the event of capsule retention, therefore, the study may not represent the whole spectrum of patients with ESRD on HD. Furthermore, the small sample size precludes recommendations regarding the application of VCE for asymptomatic HD patients. A larger clinical trial is needed.

In conclusion, this prospective cohort study is the first to use VCE to investigate the prevalence of small intestinal disease among HD patients. Although asymptomatic maintenance HD patients had a high prevalence of small bowel abnormalities (64.8\%), they did not have a high incidence of small bowel-associated events during the 1 year follow-up.

Competing interests: The study was funded by the Research Fund of Mitsukoshi Health and Welfare Foundation. The video capsule endoscopes were provided by Given Imaging Ltd. The sponsors of the study had no role in study design, data collection, data analysis, data interpretation, writing of the report, or decision to submit the manuscript for publication. Dr. Hosoe has received a research grant from Given Imaging Ltd.

\section{Institutions}

${ }^{1}$ Center for Diagnostic and Therapeutic Endoscopy, School of Medicine, Keio University, 35 Shinanomachi, Shinjuku, Tokyo 160-8582, Japan.

2 Keishinkai Tama Nagayama Jin-Naika Clinic, 1-4 Nagayama, Tama city, Tokyo 206-0025, Japan

${ }^{3}$ Department of Nephrology, Tokyo Medical University, 6-7-1 Nishi-Shinjuku, Shinjuku, Tokyo 160-0023, Japan

${ }^{4}$ Division of Gastroenterology and Hepatology, Department of Internal Medicine, School of Medicine, Keio University, 35 Shinanomachi, Shinjuku, Tokyo 160-8582, Japan

${ }^{5}$ Department of General Internal Medicine, Saitama Medical University, 38 Morohongo, Moroyama-machi, Iruma-gun, Saitama, 350-0495, Japan

${ }^{6}$ Center for Advanced IBD Research and Treatment, Kitasato University Kitasato Institute Hospital, 5-9-1 Shirokane, Minato-ku, Tokyo 108-8642, Japan.

\section{Acknowledgements}

We sincerely thank the staff of Keishinkai Tama Nagayama JinNaika Clinic.

\section{References}

1 Hallan SI, Coresh J, Astor BC et al. International comparison of the relationship of chronic kidney disease prevalence and ESRD risk. J Am Soc Nephrol 2006; 17: 2275-2284

2 Nakai S, Hanafusa N, Masakane I et al. An Overview of Regular Dialysis Treatment in Japan (as of 31 December 2012). Ther Apher Dial 2014; 18: $535-602$

3 Kang JY. The gastrointestinal tract in uremia. Dig Dis Sci 1993; 38 : 257-268

4 Etemad B. Gastrointestinal complications of renal failure. Gastroenterology clinics of North America 1998; 27: 875-892

5 Docherty E, Koulaouzidis A, Douglas $S$ et al. Use of small bowel capsule endoscopy in patients with chronic kidney disease: experience from a University Referral Center. Annals of Gastroenterology 2015; 28: $99-$ 104

6 Arakawa D, Ohmiya $N$, Nakamura $M$ et al. Outcome after enteroscopy for patients with obscure gastrointestinal bleeding bleeding: diagnostic comparison between double-balloon endoscopy and videocapsule endoscopy. Gastrointest Endosc 2009; 69: 866-874

7 Sotoudehmanesh R, Ali Asgari A, Ansari R et al. Endoscopic findings in end-stage renal disease. Endoscopy 2003; 35: 502 - 505

8 Sood P, Kumar G, Nanchal R et al. Chronic kidney disease and end-stage renal disease predict higher risk of mortality in patients with primary upper gastrointestinal bleeding. Am J Nephrol 2012; 35: 216-224

9 Saeed F, Agrawal N, Greenberg E et al. Lower gastrointestinal bleeding in chronic hemodialysis patients. Int J Nephrol 2011; 2011: 272535

10 Karagiannis S, Goulas S, Kosmadakis $G$ et al. Wireless capsule endoscopy in the investigation of patients with chronic renal failure and ob- scure gastrointestinal bleeding (preliminary data). World J Gastroenterol 2006; 12: $5182-5185$

11 Ohmori T, Konishi H, Nakamura $S$ et al. Abnormalities of the small intestine detected by capsule endoscopy in hemodialysis patients. Intern Med 2012; 51: $1455-1460$

12 Sakai E, Endo H, Taniguchi $L$ et al. Factors predicting the presence of small bowel lesions in patients with obscure gastrointestinal bleeding. Dig Endosc 2012; 25: 412-20

13 Holleran G, Hall B, Hussey $M$ et al. Small bowel angiodysplasia and novel disease associations: a cohort study. Scand J Gastroenterol 2013; 48: $433-438$

14 Kawamura H, Sakai E, Endo $H$ et al. Characteristics of the small bowel lesions detected by capsule endoscopy in patients with chronic kidney disease. Gastroenterol Res Pract 2013; 2013: 814214

15 Iddan G, Meron G, Glukhovsky A et al. Wireless capsule endoscopy. Nature 2000; 405: 417-417

16 Triester SL, Leighton JA, Leontiadis GI et al. A meta-analysis of the yield of capsule endoscopy compared to other diagnostic modalities in patients with obscure gastrointestinal bleeding. Am J Gastroenterol 2005; 100: 2407-2418

17 Saperas E, Dot J, Videla $S$ et al. Capsule endoscopy versus computed tomographic or standard angiography for the diagnosis of obscure gastrointestinal bleeding. Am J Gastroenterol 2007; 102: 731 - 737

18 Trifan A, Singeap AM, Cojocariu C et al. Small bowel tumors in patients undergoing capsule endoscopy: a single center experience. J Gastrointestin Liver Dis 2010; 19: 21-25

19 Mehdizadeh S, Chen GC, Barkodar L et al. Capsule endoscopy in patients with Crohn's disease: diagnostic yield and safety. Gastrointest Endosc 2010; 71: 121 - 127

20 von Elm E, Altman DG, Egger $M$ et al. The Strengthening the Reporting of Observational Studies in Epidemiology (STROBE) statement: guidelines for reporting observational studies. Ann Intern Med 2007; 147: $573-577$

21 Ladas SD, Triantafyllou K, Spada C et al. European Society of Gastrointestinal Endoscopy (ESGE): recommendations (2009) on clinical use of video capsule endoscopy to investigate small-bowel, esophageal and colonic diseases. Endoscopy 2010; 42: 220-227

22 Korman LY, Delvaux M, Gay G et al. Capsule endoscopy structured terminology (CEST): proposal of a standardized and structured terminology for reporting capsule endoscopy procedures. Endoscopy 2005; 37: 951-959

23 Holden RM, Harman GJ, Wang $M$ et al. Major bleeding in hemodialysis patients. Clin J Am Soc Nephrol 2008; 3: 105-110

24 Luo JC, Leu HB, Hou MC et al. Nonpeptic ulcer, nonvariceal gastrointestinal bleeding in hemodialysis patients. Am J Med 2013; 126: 264. e225-264.e232

25 Luo JC, Leu HB, Huang KW et al. Incidence of bleeding from gastroduodenal ulcers in patients with end-stage renal disease receiving hemodialysis. CMAJ 2011; 183: E1345-1351

26 Zuckerman GR, Cornette GL, Clouse RE et al. Upper gastrointestinal bleeding in patients with chronic renal failure. Ann Intern Med 1985; 102: $588-592$

27 Tsai CJ, Hwang JC. Investigation of upper gastrointestinal hemorrhage in chronic renal failure. J Clin Gastroenterol 1996; 22: 2 -5

28 Graham DY, Opekun AR, Willingham FF et al. Visible small-intestinal mucosal injury in chronic NSAID users. Clin Gastroenterol Hepatol 2005; 3: 55-59

29 Wee A, Kang JY, Ho MS et al. Gastroduodenal mucosa in uraemia: endoscopic and histological correlation and prevalence of helicobacter-like organisms. Gut 1990; 31: 1093-1096

30 Wang $X, U z u T$, Isshiki $K$ et al. Iron status and the use of non-steroidal anti-inflammatory drugs in hemodialysis patients. Ther Apher Dial 2007; $11: 215-219$

31 Endo $H$, Hosono $K$, Inamori $M$ et al. Characteristics of small bowel injury in symptomatic chronic low-dose aspirin users: the experience of two medical centers in capsule endoscopy. J Gastroenterol 2009; 44: 544 549

32 Endo H, Sakai E, Taniguchi $L$ et al. Risk factors for small-bowel mucosal breaks in chronic low-dose aspirin users: data from a prospective multicenter capsule endoscopy registry. Gastrointest Endosc 2014; 80: $826-34$

33 Watanabe T, Sugimori S, Kameda $N$ et al. Small bowel injury by lowdose enteric-coated aspirin and treatment with misoprostol: a pilot study. Clin Gastroenterol Hepatol 2008; 6: 1279-1282 
34 Sarosiek I, Selover KH, Katz LA et al. The assessment of regional gut transit times in healthy controls and patients with gastroparesis using wireless motility technology. Aliment Pharmacol Ther 2010; 31: 313 322

35 Arora Z, Parungao JM, Lopez $R$ et al. Clinical utility of wireless motility capsule in patients with suspected multiregional gastrointestinal dysmotility. Dig Dis Sci 2015; 60: 1350 - 1357
36 Francos GC, Besarab A, Joseph RE. Disorders of oesophageal motility in chronic haemodialysis patients. Lancet 1984; 28: 219

37 Siamopoulos KC, Tsianos EV, Dardamanis $M$ et al. Esophageal dysfunction in chronic hemodialysis patients. Nephron 1990; 55: 389-393

38 Dogan I, Unal S, Sindel S et al. Esophageal motor dysfunction in chronic renal failure. Nephron 1996; 72: 346-347 